\title{
Shakespeare : le poète au théâtre
}

\section{Michael Edwards}

\section{(2) OpenEdition \\ Journals}

Electronic version

URL: http://journals.openedition.org/shakespeare/1042

DOI: 10.4000/shakespeare. 1042

ISSN: 2271-6424

\section{Publisher}

Société Française Shakespeare

\section{Printed version}

Date of publication: 1 November 2007

Number of pages: 121-130

ISBN: 2-9521475-3-1

Electronic reference

Michael Edwards, «Shakespeare : le poète au théâtre ». Actes des congrès de la Société française Shakespeare [Online], 24 | 2007, Online since 30 March 2010, connection on 01 May 2019. URL : http:// journals.openedition.org/shakespeare/1042 ; DOI : 10.4000/shakespeare.1042

This text was automatically generated on 1 May 2019.

(c) SFS 


\title{
Shakespeare : le poète au théâtre
}

\author{
Michael Edwards
}

\section{I}

1 « Now spurs the lated traveller apace, / To gain the timely inn » (Macbeth, III.iii.6-7). Cette phrase n'a rien de particulièrement "poétique », mais elle vibre de poésie; elle paraît négligeable, mais dans la situation théâtrale elle est étonnante. Elle est prononcée par le Premier Meurtrier lorsqu'il attend Banquo pour le tuer. Un malfaiteur peut, malgré la concentration de son esprit sur l'acte à accomplir, prendre conscience de la réalité de l'heure, de ce qui, dans le jour finissant, ne le touche pas personnellement; il peut songer à quelqu'un d'autre, et surtout se mettre à sa place, en imaginant le plaisir qu'aura le voyageur qui se trouve bien tard sur la route à voir enfin une auberge devant lui, « timely », au bon moment. Le Meurtrier agit, à bien y réfléchir, comme Shakespeare, qui semble, à tout instant, penser à tout (d'où sa grande différence avec Racine et l'hésitation de beaucoup de lecteurs français), et qui entre, non pas de temps en temps mais inlassablement, dans la peau des autres. Même si l'on décide que c'est son subconscient qui suggère au Meurtrier ce petit récit d'un homme échappant au danger - comme il aimerait lui-même, le meurtre commis, se trouver en sécurité - on découvre le désir de Shakespeare de rendre présent l'être même, la profondeur secrète, d'un personnage tout à fait secondaire. Si on accepte que le Meurtrier parle sans arrière-pensée, on s'aperçoit qu'en passant dans l'esprit d'un assassin, puis, grâce à l'intérêt curieusement désintéressé de celui-ci, dans l'esprit d'un voyageur surpris par la nuit, anxieux, puis rassuré, Shakespeare se hasarde doublement dans le je des autres et offre un petit aperçu de sa création poétique et théâtrale.

2 Sortir ainsi du moi est un vrai choix de poète ; le faire à l'aide d'un meurtrier est la marque d'une sorte de charité poétique extraordinaire, qui s'exerce avant tout, dans cette pièce, dans la création de Macbeth. Lorsque Macbeth entre pour la dernière fois sur scène et rencontre Macduff, que ses premiers mots sont complexes ! 
Of all men else I have avoided thee :

But get thee back, my soul is too much charged

With blood of thine already. (v.viii.4-6)

3 Macbeth a évité Macduff à cause de l'avertissement de la Première Apparition, mais on doit sentir aussi dans la voix de l'acteur une autre raison, plus généreuse. Macbeth, ayant fait tuer la femme et les enfants de Macduff, ne veut pas le tuer, lui, à cause d'un reste de compassion que Shakespeare lui reconnaît. Il est vrai que, s'il sait son âme "chargée ", alourdie, par le meurtre, il la sait aussi "accusée »: il hésite à ajouter à sa charge une autre mort. Mais le fait même de penser au jugement des autres - voire au Jugement dernier - surprend chez celui qui vient de réduire la vie, à la scène $\mathrm{v}$, à un conte débité par un idiot et ne signifiant rien. Shakespeare, en faisant parler l'autre et, dans ce cas précis, en écoutant les paroles d'un homme entièrement étranger à sa propre manière d'être, semble chercher, au-delà de l'immense complexité de ce que Macbeth lui-même appelle l'âme, toutes les raisons de ne pas le condamner de façon catégorique. La survie chez Macbeth d'un sens de l'univers moral et d'une volonté, même très partielle, de ne pas nuire, sert le dessein global de Shakespeare, sa recherche de toutes les ressources qui permettront à la tragédie de dépasser la catastrophe. Elle témoigne aussi de son désir de comprendre, en la vivant dans la poésie du théâtre et dans le théâtre de la poésie, la lointaine altérité de l'autre.

Il ne s'agit pas seulement de psychologie - bien que la psychologie de ses personnages importe à Shakespeare, comme un ensemble de détails qui définit la différence de l'autre et qui révèle progressivement son être, les choix existentiels et ontologiques par lesquels il est ce qu'il est. Pour le poète, qui est tenté sans cesse de parler en son propre nom sans chercher la vérité transpersonnelle sur laquelle donne sa propre expérience, le théâtre peut paraître l'occasion de renoncer au lyrisme du moi et de s'aventurer dans le je des autres. Il permet à Shakespeare de renouveler l'acte poétique, en faisant proliférer le je qui écrit et le je qui parle, cette multiplicité de je dans une abondance de personnages correspondant à la vision dans son théâtre de la foison du réel, de l'exubérance à la fois redoutable et prodigieuse des choses à comprendre et à organiser.

\section{II}

5 On entrevoit ici un début de réponse à la question : Pourquoi Shakespeare, poète, écrit-il des pièces de théâtre? Le théâtre transforme la poésie en parole, et peut devenir la recherche de la parole de l'autre. En un sens, la question, formulée ainsi, est maladroite : elle pourrait faire croire à une distinction entre poésie et théâtre, en accordant à la poésie la prééminence. Car, sans en être entièrement conscients, nous avons tendance à renverser, simplement, les priorités de la poétique classique, telle qu'elle est énoncée, de façon brutale mais claire, dans le Dictionnaire universel de Furetière de 1690, au mot poème. $\mathrm{Au}$ lieu de croire, comme lui, que les « vrais » poèmes sont les épopées et les tragédies et que les «vers lyriques » - par exemple, les sonnets - ne méritent le nom de poème que "fort abusivement", il est séduisant de supposer, au contraire, que seule la poésie "lyrique » est véritable, étant seule capable de créer chez le lecteur une expérience très spéciale que nous avons appris à appeler poétique. Dans une telle perspective, le choix de Shakespeare peut paraitre troublant. Il suffit de changer de perspective, cependant (s'il en est besoin), pour voir que la question à poser n'est pas de savoir comment Shakespeare réussit malgré tout, malgré les entraves du théâtre, à œuvrer en poète, mais comment le 
travail au théâtre du plus grand poète anglais peut élargir notre sens de la poésie et de son possible.

6 Afin d'avancer, je continuerai d'écouter la dernière scène de Macbeth selon l'in-folio de 1623, qui devient dans beaucoup d'éditions modernes une série de scènes. Je choisis à dessein un moment du théâtre shakespearien où un ordre accompli se déclare dans le déroulement d'une action apparemment désordonnée et certainement tumultueuse, et où se trouve peu de poésie selon les définitions modernes (ou anciennes) du mot, le quasimonologue de Macbeth: "Tomorrow, and tomorrow, and tomorrow", étant déjà intervenu à la scène v. Cela me permet aussi de réfléchir sur un personnage sans grande importance : Siward, et de noter le fait évident que les personnages au théâtre ne sont pas seuls, et que le théâtre accroît également le possible de la poésie par la présence des personnages les uns pour les autres, par des paroles qui se disent dans des situations déterminées.

7 Siward ne paraît qu'à la fin, mais il est présenté dès l'acte III par une série d'épithètes. Il entre progressivement dans la conscience du spectateur comme "warlike Siward» (III .vi.31), «Old Siward» (Iv.iii.134), « good Siward » (Iv.iii.190), pour que Shakespeare attire l'attention, discrètement, sur un rôle secondaire qui donnera, au dénouement de la tragédie, toute la mesure de sa valeur. Lorsqu'il est présenté finalement comme l'oncle de Malcolm : «His uncle Siward » (v.ii.2), ne croyons pas que ce lien de parenté soit moins important que les diverses indications de son expérience et de son caractère. Si son rôle dans l'action de la pièce est d'apporter à Malcolm le secours des soldats anglais, son rôle sur la scène, pour ainsi dire, est de réagir à la mort de son fils. Les questions laconiques qu'il pose à Ross: "Then he is dead? " et « Had he his hurts before? ", font penser d'abord à une sorte de stoïcisme, émouvant mais un peu refroidissant aussi. Ross l'ayant assuré, cependant, que son fils fut tué face à son adversaire, Siward dit ceci :

Why then, God's soldier be he !

Had I as many sons as I have hairs,

I would not wish them to a fairer death :

And so, his knell is knolled. (v.ix.13-16)

8 Tout ce qu'il y a d'admirable dans ce passage vient, non pas de l'Histoire d'Angleterre de Holinshed, mais de Shakespeare, et en premier lieu le jeu de mots surprenant entre « hairs » (cheveux) et « heirs" (héritiers). Shakespeare semble s'intéresser d'abord à la psychologie du langage, au fait qu'au paroxysme de l'émotion, l'esprit se meut très rapidement (ou pas du tout) et s'avère capable d'appréhender des relations en même temps entre les mots et entre les sens. C'est un intérêt de poète, qui peut considérer que toute poésie est un jeu - un jeu parfaitement grave - de mots et de sens, et que dans l'acte poétique les choses les plus importantes arrivent parfois en une fraction de seconde et avant que l'on ait le loisir d'y penser. Mais il s'intéresse surtout, me semble-t-il, au fait que Siward, en observant le rapport entre l'expression consacrée : «autant qu'il y a de cheveux sur la tête ", et son fils et héritier auquel il s'apprête à l'appliquer, s'élève audessus de son émotion personnelle et atteint une sorte d'impersonnalité, grâce à laquelle il regarde la situation à la fois selon la vérité de sa perte et selon la vérité générale de la vie sous le signe du courage et du sacrifice. Le moment est sans doute très anglais, le calembour (qui sert aussi, peut-être, à libérer par le grotesque une émotion insupportable) ayant pour effet, non pas de diminuer mais d'augmenter la force de la réplique. Et c'est la poésie qui lui permet à nouveau de se ressaisir, lorsqu'il se dit, dans un autre demi-vers, que le «knell» de son fils «is knolled». Il emploie deux mots 
apparentés, mais ce qui serait, dans un autre contexte, une maladresse, s'entend ici comme une manière impersonnelle et définitive de dire qu'une vie est finie.

Malcolm, qui est jeune et qui n'a pas d'enfants, ne comprend ni la fermeté ni, si je puis dire, la poésie de Siward. Il l'accuse de ne pas être suffisamment touché par la mort de son fils, qui «mérite plus de chagrin ». (Je note, en partie pour confirmer la capacité de Shakespeare de songer à beaucoup de choses en même temps, qu'il a déjà fait sentir le manque d'expérience de Malcolm, sa réaction purement théorique devant la présence de l'autre et devant les façons de se conduire dans la vie, au moment où Macduff se lamentait de la perte de sa femme et de ses enfants et où Malcolm le blâmait, au contraire, de ne pas "prendre cela comme un homme " (Iv.iii.220). C'est une des petites touches par lesquelles Shakespeare laisse entendre que le renouveau effectué par la tragédie ne sera pas idéal, que les problèmes du réel continueront, que l'Écosse, sauvée, pourra souffrir de l'imperfection du nouveau roi.) Et c'est à Siward de montrer à Malcolm comment se situer parfaitement par rapport à l'être d'autrui :

He's worth no more ;

They say he parted well and paid his score :

And so, God be with him ! (v.ix.17-19)

Le jeune Siward ne mérite pas plus de chagrin, puisque dans la lumière absolue où il se trouve maintenant, on ne peut rien mériter, ayant simplement une dette à payer; il a bien payé la sienne envers la nature, en rendant la vie qu'elle lui avait donnée. Ce qui ne signifie pas que le père, une fois seul, ne pleurera pas son fils pour une raison tout autre et évidente. Pour le moment, il tâche de voir son fils selon la vision la plus élevée des choses dont il est capable, et la rime («more/score») - qui est l'œuvre strictement de Shakespeare, qui l'offre à Siward - lui permet de donner une forme également absolue à sa pensée.

Suivent des mots d'une simplicité extrême, mais qu'il suffit d'entendre au théâtre, dans le contexte de la scène, pour en éprouver la grandeur : «And so, God be with him! " La poésie, et singulièrement celle de Shakespeare, est aussi la recherche de paroles qui comptent : "I am a very foolish, fond old man ", «Sir John and all», «I melt, and am not ", et ainsi de suite. Ici, l'émotion de Siward - évidemment profonde et qui le secoue terriblement - s'exprime par un salut familier à l'époque de Shakespeare : «God be with you ", transposé et rendu à son sens exact. Arrivé au terme de son premier travail de deuil, Siward souhaite pour son fils, qu'il ne peut plus aider, que Dieu soit littéralement avec lui. Il dit ce qu'il pense, et Shakespeare aussi, je crois, au moment où le monde se délivre de Macbeth par ce que Malcolm est sur le point d'appeler, au moyen d'une autre tautologie éloquente, « la grâce de la Grâce ».

12 Nous savons qu'il n'existe pas de poésie dans le théâtre de Shakespeare au sens où il arrêterait tout afin de s'exprimer en écrivant de beaux vers. Il faut ajouter, cependant, que si le théâtre transforme toute poésie en parole et qu'il place toute parole dans une situation - en obligeant la poésie à s'incarner, en quelque sorte, dans la vie des hommes c'est pour que la poésie gagne à paraître sur la scène. Les mots les plus ordinaires, comme les derniers cités de Siward, peuvent résonner de toute l'émotion, toute la pensée, d'une pièce. Il convient d'ajouter aussi que, si la poésie se trouve partout dans une pièce de Shakespeare, en touchant chaque syllabe et en s'accomplissant dans la pièce entière considérée dans son ensemble, ses moments forts peuvent survenir n'importe quand et chez les personnages les plus inattendus. 


\section{III}

13 À observer Siward, on se rend compte aussi que chaque pièce de Shakespeare est un poème qui lui permet de multiplier les points de vue avant de chercher à les ordonner. Chaque pièce est une mise en œuvre de la " pensée » de Shakespeare, qui est pleinement présent dans le moindre détail. Et cette ordonnance n'est pas nécessairement à découvrir dans un réseau d'images, comme le voulait jadis une certaine approche de Shakespeare, pourtant originale et éclairante, qui procédait d'une réflexion, disons, post-romantique sur la poésie.

14 Je pense plutôt à quelques mots que Siward ajoute aux vers que je viens de citer, et qui sont les derniers qu'il prononce : "Here comes newer comfort ». Il voit Macduff avec la tête de Macbeth, et ses paroles peuvent sembler fonctionnelles, simple transition à ce qui suit. Le mot « comfort » devrait éveiller, cependant, notre mémoire. Dans les batailles qui ouvrent la pièce, la victoire de Macbeth sur Macdonwald, qui apporte du « comfort ", du réconfort, à l'armée du roi, est suivie aussitôt par le "discomfort » représenté par un nouvel assaut des troupes norvégiennes (I.ii.27-8). Le mot fait sentir, dès le début, l'idée de force, d'encouragement vigoureux, qui le sous-tend mais qu'il a un peu perdu en anglais moderne. Il revient au moment où Ross retrouve Malcolm et Macduff en Angleterre et parle de la détresse des Écossais. « Nous y allons, répond Malcolm, que ce soit leur réconfort! » (IV.iii.188-9). Il est à remarquer que Siward, que Malcolm mentionne aussitôt, avec les dix mille soldats qu'il commande, participe de ce "comfort». Ross regrette, cependant, de ne pas apporter de « comfort » à Macduff, qui apprend par lui le massacre de sa famille et qui, malgré l'injonction de Malcolm, refuse d'être " comforted ", de se laisser réconforter et de prendre courage. Il se désole et se concentre sur son désir de vengeance, par une réaction tout à fait naturelle et émouvante à sa façon.

La scène où Siward apprend la mort de son fils est liée à cette autre scène, non seulement par son contenu mais par le fait que c'est de nouveau Ross qui apporte la nouvelle et qui, à chaque occasion, hésite avant de parler. Ce détail n'a pas, si je l'ai bien compris, une signification particulière, il n'influe pas sur l'interprétation des deux scènes. Il contribue plutôt à cette écriture, pour ainsi dire, musicale de Shakespeare, par laquelle il satisfait son aspiration, qui est aussi la nôtre, à la forme : à des motifs, à des harmonies, qui se tiennent en dehors de l'explicable et qui ne suppriment pas les circonstances du hasard, l'autonomie du réel.

On voit la beauté de la petite phrase et la raison pour laquelle cette scène est préparée par celle de Macduff, en réfléchissant à la merveille du ressaisissement de Siward. Au moment même où il a un besoin urgent de réconfort, il réagit, d'une manière qui n'est pas du tout naturelle, en pensant au réconfort que la mort de Macbeth apporte à l'Écosse. Pendant un moment - quelques secondes - notre attention se fixe sur l'être remarquable de Siward, capable de sortir de lui-même lors d'une telle crise et de reconnaître ce qui importe plus que son déchirement personnel insoutenable. Même le mot "newer » exprime l'effort qu'il fait. L'étrange comparatif ne signifie pas, comme il le devrait, que ce réconfort-là soit " plus nouveau »; il suggère, de manière elliptique, que ce nouveau réconfort est, disons, plus grand, plus réjouissant que celui dont il a besoin.

Le mot comfort surgit de la pièce; il gagne ainsi en profondeur même quand il est prononcé par un personnage secondaire. On pense au mot fear, qui rôde dans Macbeth, qui 
semble être toujours prêt à paraître et qui hante en particulier le personnage principal. Le mot « occupe l'esprit » de Macbeth, comme dirait Baudelaire (voir « Au lecteur » dans Les Fleurs du mal), au point où il modifie dans sa mémoire, sous son influence, les avertissements des Apparitions évoquées par les Sorcières : il croit se souvenir qu'elles lui avaient dit de «fear » qui n'est pas né d'une femme (v.vii.4) et de « Fear not » avant que le bois de Birnam ne vienne à Dunsinane (v.v.44-5). Dès que les Sorcières lui eurent annoncé qu'il serait roi, il fut saisi d'une peur incontrôlable, visible par Banquo (I.iii.51), en voyant sans doute le mal qui sommeillait en lui et en découvrant le Mal même, la condition terrible d'un monde déchu. Comme nous entendons chez Macbeth une parole impersonnelle, ou qui dépasse l'individu - une voix profonde qui dit la peur de ce que peut un monde désaxé et la peur des forces étrangères qui l'habitent - nous entendons une voix profonde qui parle par Siward de l'existence du comfort, d'un réconfort, d'une force source de courage qui constitue, à bien y penser, le contraire de la peur et une réponse au mal.

Cet autre surcroît de poésie rendu possible par la narration théâtrale est l'affaire à la fois du personnage principal et d'un rôle secondaire, sinon tertiaire. Et le fait que Siward a si peu d'importance dans la pièce rend précieux ce qu'il lui apporte, l'exemple d'un homme plongé dans la douleur mais qui se soucie du bien public - dans un pays, du reste, autre que le sien. En s'investissant ainsi dans la réalité et dans les paroles de tant de personnages, quel que soit leur rang dans l'action, Shakespeare suggère l'importance de chacun, dans une optique chrétienne ou humaniste ; il s'oblige aussi à sortir de lui-même, à écouter l'autre, par une attention constante qui me semble, je l'ai dit, profondément poétique. Mais surtout, la "pensée » de la pièce a besoin de Siward pour se compléter comme elle a besoin de Cawdor. Cawdor n'est même pas un personnage, et n'existe que dans les paroles des autres, qui le présentent comme «ce plus déloyal des traîtres » (I .ii.53). Il n'a qu'à mourir par ordre du roi, mais voilà qu'il «avoue» ses trahisons, demande «pardon » et fait preuve d'un " profond repentir » (I.iv.5-7). Ce retournement est parfaitement inutile à l'action de la pièce et particulièrement nécessaire, par conséquent, à son effet total, comme l'est aussi, à l'effet de King Lear, la confession tout à fait imprévue de Goneril, qui avoue, dans les coulisses, avoir empoisonné sa sœur. Il ne s'agit pas exactement de morale, et cette multiplicité de détails ne contribue pas précisément à une structure intellectuelle, à une Pensée à cerner et à rendre explicite. Chaque pièce est un poème tremblant de toutes les contradictions de la vie (si Shakespeare invente, dans King Lear, la confession de Goneril et la repentance d'Edmund, il invente aussi la mort de Cordelia), et c'est au spectateur de chercher, devant la prolifération des notes diverses et devant le mouvement fondamental de la pièce, non pas tellement sa signification, mais dans quel sens elle va. Chaque pièce est un acte poétique de Shakespeare, qui a la générosité non seulement de laisser parler une grande variété de personnages, en donnant à chacun sa chance, mais de seulement suggérer le point de vue qui pourrait être le sien, sans l'imposer. D'où l'invitation au spectateur de se mettre en présence de tous les personnages et de tout écouter.

IV

Le travail de Shakespeare au théâtre, comme celui d'autres dramaturges-poètes, consiste, sous cet angle, à diriger la poésie vers la parole. Et pour en saisir l'importance, on n'est pas obligé de croire que le langage, et singulièrement le langage en poésie, soit d'abord un 
moyen de s'exprimer et de communiquer avec autrui. On peut très bien le tenir, en premier lieu, pour le don inestimable de nommer les êtres et les objets du monde ambiant, de les mettre en rapport avec nous-mêmes, et d'envisager leur possible dans le changement qu'opèrent sur eux les sons, les rythmes, la mémoire, les mots, et la conscience humaine qui se meut ainsi entre les mots et les choses. La poésie n'est pas un langage spécial, mais un enrichissement du langage, la mise en œuvre, et la mise en évidence, de toute la vie secrète des mots, de tout ce qui se trouve en deçà ou au-delà du sens. C'est du reste Shakespeare qui est le plus apte à nous en convaincre, comme à nous confirmer le pouvoir de la poésie, sinon de changer le réel, du moins de renouveler en profondeur notre perception du réel et de nous-mêmes. La vertu recréatrice de sa poésie (dont je me suis abstenu de parler dans une méditation où j'ai préféré trouver la poésie là où on ne la cherche pas d'habitude) est si immense qu'il sent peut-être le besoin de tout soumettre au concret des échanges humains. La puissance avec laquelle il imagine le monde à nouveau, et ne cesse de le faire, trouve une sorte de légitimation dans la spécificité ontologique de ses personnages, dans les situations existentielles toujours en mouvement. Cet enracinement d'une grande vision dans le temps et les lieux du vécu, dans des mots prononcés par des gens pris dans la trame des événements, est un avantage pour Shakespeare, et je note finalement que la poésie au théâtre n'est pas signalée : à moins que des circonstances particulières ne le demandent, elle ne paraît pas, par exemple, sous forme strophique. La poésie peut ainsi s'insinuer dans la vie que le théâtre présente. La vie devient poésie, sous nos yeux.

\section{ABSTRACTS}

Why should the greatest English poet have written essentially for the theatre? In part because theatre directs poetry into speech, and can become thereby a search for the speech, the inner language, of the other. The abundance of Shakespeare's characters enables him not only to multiply the points of view that need to be brought into order, but also to forget and to extend himself in listening to the depths of numerous ways of being, his genius being to attend as closely to the inner life of secondary characters, such as Siward or the First Murderer in Macbeth, as to that of the principals. The study of his poetry-in-theatre can greatly extend our generally rather narrow modern notions of what poetry is.

La question fondamentale à poser : pourquoi Shakespeare, poète, choisit-il le théâtre ? Quelques débuts de réponse: le théâtre lui permet de renouveler entièrement l'acte poétique, en démultipliant le moi qui écrit et le je qui parle; le théâtre accroît le possible de la poésie, en particulier par la présence des personnages les uns pour les autres, et par le silence; chaque pièce devient un poème de Shakespeare, grâce, non pas à des réseaux d'images, mais à la présence de Shakespeare - de sa «pensée »- dans le moindre détail. 


\section{AUTHOR}

\section{MICHAEL EDWARDS}

Études à Cambridge ; carrière universitaire en Angleterre ; enseignement depuis 2003 au Collège de France (Chaire d'Étude de la création littéraire en langue anglaise). Dernier recueil de poèmes : Rivage mobile (Arfuyen, 2003) ; dernier livre des proses : Un monde même et autre (Desclée de Brouwer, 2002). Parmi ses livres critiques récents, trois études sur Shakespeare : Shakespeare et la comédie de l'émerveillement (Desclée de Brouwer, 2003), Racine et Shakespeare (PUF, 2004), Shakespeare et l'œuvre de la tragédie (Belin, 2005). 